Historic, Archive Document

Do not assume content reflects current scientific knowledge, policies, or practices. 


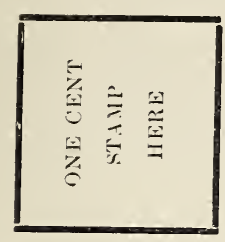

Vinnennes 7/unstericas

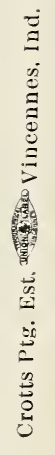

$\sum$ 


\section{Vincennes Nurseries}

॥W. C. REED \& SON, Proprietors General Nursery Stock

Phone 196 BULLETIN No. 1 Dec. 20, 1920

List of Stock in Storage for early shipment.

Terms and Conditions same as Fall Trade List. All Stock has been carefully graded.

\section{Please Sibmit List of Your Wants for Prices.}

\section{APPLE-Two Year Grafts.}

The three smaller grades are largely whips cut back at one year, being mostly One Year with extra fine root system.

\begin{tabular}{|c|c|c|c|c|c|}
\hline $\begin{array}{r}11-16 \\
5-7 \mathrm{ft} \\
\ldots .200\end{array}$ & $\begin{array}{c}9-16 \\
46 \mathrm{ft} \\
150\end{array}$ & $\begin{array}{r}7-16 \\
4-5 \mathrm{ft} \\
450\end{array}$ & $\begin{array}{l}3-4 \mathrm{Ft} . \\
620\end{array}$ & $\begin{array}{l}2-3 \mathrm{ft} \\
825\end{array}$ & $\begin{array}{l}18-24 \\
\text { inch } \\
130\end{array}$ \\
\hline Delicious $\quad \ldots \ldots \ldots \ldots \ldots \ldots 80$ & & 222 & 674 & 470 & 195 \\
\hline Grimes Golden . . . . . . . . 590 & & 15 & 480 & 300 & 85 \\
\hline Tonathan $\ldots \ldots \ldots \ldots \ldots$ & & 950 & 280 & 625 & 88 \\
\hline 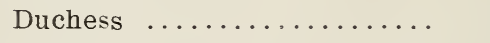 & 200 & 75 & 80 & 574 & 200 \\
\hline Staymans Wine Sap .......235 & 130 & 40 & & & \\
\hline Wealthy $\ldots \ldots \ldots \ldots \ldots$ & & 410 & 308 & 240 & 120 \\
\hline Stark .............. 60 & 125 & 160 & 50 & 46 & 6 \\
\hline olden Sweet ..........100 & $277^{-}$ & 247 & 53 & 128 & 30 \\
\hline Ensee $\ldots \ldots \ldots \ldots \ldots \ldots$ & 64 & 208 & 40 & 130 & 20 \\
\hline Wolf River ............ 50 & 38 & 74 & 20 & 24 & \\
\hline$\ldots \ldots \ldots \ldots \ldots \ldots 17$ & 33 & 63 & 40 & 30 & \\
\hline Ben Davis .............. & 25 & 12 & & 5 & \\
\hline Regans Red (B. B. Davis)... 10 & 44 & 100 & & & \\
\hline etts $\ldots \ldots \ldots \ldots \ldots \ldots$ & 30 & 120 & 10 & 14 & \\
\hline Ingram $\ldots \ldots \ldots \ldots \ldots \ldots \ldots$ & 67 & 93 & 90 & 52 & $2 s$ \\
\hline Red Astrachan .......... 11 & & 7 & & 140 & 20 \\
\hline Sweet June ............ 23 & 9 & 20 & & & \\
\hline ni $\ldots \ldots \ldots \ldots \ldots \ldots, 7$ & 18 & 77 & & 20 & \\
\hline ango $\mathrm{Stb} \ldots \ldots \ldots \ldots .30$ & 65 & 80 & & & \\
\hline en Blush ............ & 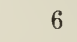 & 71 & 128 & 4 & 10 \\
\hline York Imperial $\ldots \ldots \ldots \ldots \ldots$ & & 7 & 24 & 22 & 10 \\
\hline er Banana............. & & & 25 & 10 & \\
\hline Rambo $\ldots \ldots \ldots \ldots \ldots$ & & & 30 & 23 & \\
\hline David .......... & & & 69 & 40 & 20 \\
\hline$R \in d \ldots \ldots \ldots \ldots$ & 10 & 25 & 7 & 15 & \\
\hline e Beauty ............ & & 280 & 53 & 20 & \\
\hline an Sweet...$\ldots \ldots \ldots$. & 40 & 83 & 30 & 40 & \\
\hline scent $\ldots \ldots \ldots \ldots \ldots$ & 20 & 43 & 20 & 40 & \\
\hline 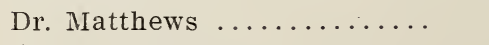 & 20 & 60 & 36 & 50 & \\
\hline Yel Transparent .......... & & 245 & 185 & 600 & \\
\hline Turley (New very fine)... & & 110 & 35 & 20 & \\
\hline Carson (New Ea Summer) & & & 77 & 68 & 13 \\
\hline
\end{tabular}

Turley One Year Buds, 300-5-6 ft.; 200-4-5ft.; 200-3-4 ft. Carson One Year Buds, 100-5-6 ft.; 100-4-5 ft.; 100-3-4 ft. Yellow Transparent, 15004 to $5 \mathrm{ft}$. One Year.

York Imperial, 5004 to $5 \mathrm{ft}$. One Year. 
STANDARD PEAR-One Year.

Keiffer

Garber $150 \quad 190 \quad 128$

Bartlett 227128

Duchess $100 \quad 100$

Koonce

$100 \quad 100$

Wilder $100 \quad 100$

Clapps $100 \quad 100$

PLUM ON PEACH-One Year.

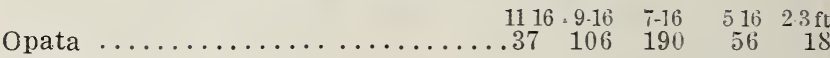

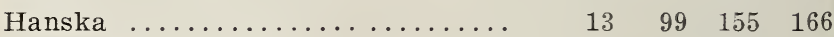

Wachampa ...............65 $148 \quad 196 \quad 125 \quad 51$

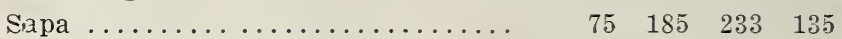

Wild Goose ................ $27 \quad 27$

Red June ................. $20 \quad 50 \quad 20$

Burbank .................. $185 \quad 400$

\section{GOOSEBERRY AND CURRANTS.}

10650 Downing XX One Year equal to 2 year@

8650 Downing No. 1 One Year.

1900 Downing No. 2 One Year for Transplanting

2400 Houghton XX One Year very fine

600 Houghton No. 1 One Year

2150 Blk. Currants XX One Year

$20 \mathrm{co}$ Concord

GRAPES-One Year.

1200 Niagara

\section{SHRUBEERY.}

1000 Spiraet Van Houtte 2 to $3 \mathrm{ft}$. heary @

1000 Spiraet Van Houette 18 to 24 inch@

200 Altheas strong bushes @

100 Altheas asst. 2 to 3 ft. @

1000 Geıman Iris@

1000 Japan Barberry 18 to 24 inch

50 C Japan Iris 12 to 18 inch

200 Lilacs Common 3 to 4 and 4 to $5 \mathrm{ft}$.

\section{GARDEN ROOTS.}

500 Rhubarb XX 2 year @

1000 Asparagus strcng 2 year@

\section{NUT' TREES.}

Grafted Pecans, leading Northern varieties.

3 to 4 fret @ per 100

4 to5 feet@per100

5 to 6 feet $@$ per 100

6 to 8 feet @ per 100

8 to 10 feet@per 100

10 to 12 feet@per 100

Seedling Pecans 2 to 3 feet@per100

Seedling Pecans 3 to 4 feet @ per 100

Seedling Pecans 4 to 5 feet @ per 100

Seedling Pecans 5 to 6 feet @ per 100

Seedling Blk. Walnuts 4 to 5 feet@

Seedling Blk. Walnuts 5 to 6 feet@

Japan Walnut 3 to 4 feet @

Japan Walnut 4 to 5 feet @ 